\title{
Performance of Four Malaria Rapid Diagnostic Tests (RDTs) in the Diagnosis of Malaria in North Central Nigeria
}

\author{
Ijezie Ntomchukwu Simon ${ }^{1,}$, Matur Bernard Malau ${ }^{2}$, Malann Yoila David ${ }^{2}$, Njab Jean Emile ${ }^{3}$ \\ ${ }^{1}$ National Malaria Elimination Programme, Federal Ministry of Health, Abuja, Nigeria \\ ${ }^{2}$ Department of Biological Sciences, University of Abuja, Abuja, Nigeria \\ ${ }^{3}$ TQMLAB Project for Africa, Abuja, Nigeria
}

Email address:

ijeziesimon@yahoo.com (I. N. Simon), malaumatur@gmail.com (M. B. Malau), malannyd@gmail.com (M. Y. David), jeanonjab@gmail.com (N. J. Emile)

${ }^{*}$ Corresponding author

\section{To cite this article:}

Ijezie Ntomchukwu Simon, Matur Bernard Malau, Malann Yoila David, Njab Jean Emile. Performance of Four Malaria Rapid Diagnostic Tests (RDTs) in the Diagnosis of Malaria in North Central Nigeria. International Journal of Infectious Diseases and Therapy.

Vol. 5, No. 4, 2020, pp. 106-111. doi: 10.11648/j.ijidt.20200504.11

Received: August 27, 2020; Accepted: September 14, 2020; Published: October 7, 2020

\begin{abstract}
The study on the performance of four rapid diagnostic test (RDT) kits (Global, LabAcon, SD Bioline and CareStart kits) in the diagnosis of Plasmodium falciparum was carried in North Central Nigeria for a period of twelve months to evaluate the performance of the kits using samples of symptomatic patients attending clinic. The performance of the kits was compared with that of microscopy as standard. Result of the sensitivity of the four RDT kits revealed that Global, LabAcon, SD Bioline and CareStart recorded $86.50 \%, 84.90 \%, 86.50 \%$ and $83.70 \%$ respectively while their level of specificity was $95.40 \%, 95.30 \%, 95.80 \%$ and $96.00 \%$ respectively. The four kits recorded no significant difference in sensitivity and specificity $(\mathrm{p}>0.005)$. SD Bioline, however, demonstrated the highest accuracy of $92.90 \%$ while LabAcon had the lowest accuracy $(92.10 \%)$. The positive predictive values and negative predictive values of the four kits were; Global $(87.80 \%$ and 94.10\%), SD Bioline (87.80\% and 94.30\%), LabAcon $(86.20 \%$ and $94.10 \%)$ and CareStart $(85.00 \%$ and $94.60 \%)$. There was no significant difference in either the accuracy, positive predictive value and negative predictive value of the four kits $(p>0.005)$. The overall performance of the four kits was also insignificantly different $(p>0.005)$. The performance of the four kits was statistically different compared with microscopy test $(\mathrm{p}<0.005)$, so the RDT kits cannot replace microscopy, being the gold standard but can, however, be used for malaria diagnoses for ease of analysis.
\end{abstract}

Keywords: Sensitivity, Specificity, Malaria, Accuracy, Predictive Value

\section{Introduction}

Malaria presents a diagnostic challenge in most resource poor areas where malaria is epidemic. Within regions like that, the diagnosis of malaria is always done clinically with attendant inaccuracy. Laboratory diagnosis of malaria is essentially necessary for proper treatment decision. Microscopy, involving the examination of thick and thin blood under the microscope, is the age long method of diagnosing malaria.

Malaria microscopy requires considerable skill, intensive labor and time leading to longer turn-around-time. Irrespective of these shortcomings, it is still the gold standard for diagnosis of malaria both in medical practice and research [1]. Microscopy, though the gold standard, is highly dependent on the microscopist. Therefore, to maintain standard and quality, initial training and continuous retraining are needed both of which are challenging to maintain in poor countries where malaria is endemic. Lack of training and retraining on malaria microscopy has led to over diagnosis of malaria using this method [2]. Malaria Rapid Diagnostic Test kits were, therefore, developed and is being used in many countries to reduce the deficiencies of microscopy [3]. 
The development of rapid diagnostic tests (RDTs) amongst other different methods was born out of the need for a simple, sensitive diagnostic test for malaria. The use of RDTs at first met strong opposition by the malaria community because of its cost. However, some of reports from program managers have recognized that RDTs may have their place since expert microscopy in malaria- endemic countries is hard to establish and cost of RDTs has reduced significantly. Further, parasitological diagnosis is a prerequisite for treatment with artemisinin based combination therapy (ACT). Providing parasitological confirmation at all levels of health care, however, presents a strict challenge. Though expanding the use of microscopy is a potential answer but the cost and logistic challenges in rural areas limits the use of microscopy to hospitals and well equipped laboratories. Reading a malaria slide accurately takes a lot of skill and years of experience.

\section{Materials and Methods}

\subsection{Study Area}

The study was conducted in three states of the North Central Geopolitical zone (Nasarawa, Benue, and Niger) and the Federal Capital Territory (FCT) of Nigeria. Federal Medical Center (FMC) Keffi, Federal Medical Center (FMC) Makurdi, General Hospital Suleja and Maitama General Hospital were points of sample collection in Nasarawa, Benue, Niger states and FCT respectively. FMC Keffi, and FMC Makurdi are tertiary health care institutions while General Hospital Suleja and Maitama General Hospital are secondary health care institutions. Owing to the size, specialized nature and location of these hospitals, patients attend clinic there from all over the various states and even neighboring states.

\subsection{Study Population}

Samples were collected from symptomatic patients visiting these major Government hospitals in each of the states and FCT. Only symptomatic patients consulted by the doctor and suspected of having malaria were included. There was no age restriction. Malaria test was requested by the doctor for patients to be included. A total of 250 samples were collected in each state making a cumulative total of 1000 samples. Ethical approvals were obtained from the ethical committees of the various hospitals and only patients who consented were included in the study. The samples were tested using each of the four kits and microscopy.

\subsection{Sample Collection and Preparation}

$4 \mathrm{ml}$ of venous blood was collected into sample bottle containing potassium EDTA anticoagulant.

Both thick and thin films were made from each patient's sample immediately at the point of collection on same slide as adopted by Chesbrough [4].

A labeled grease free and clean microscope slide was placed on a slide template. $2 \mu \mathrm{l}$ of blood was placed at the center of the slide for thin film, and another $6 \mu$ l of blood placed about $15 \mathrm{~mm}$ to the right. Immediately the thin film was spread using a smooth edged glass spreader and the thick smear was spread to cover an area of about $10 \mathrm{~mm}$ diameter. The films were allowed to air dry in a horizontal position on a flat surface and the thin film was fixed with absolute methanol for 2 minutes while the thick film was heat fixed at a temperature of $40^{\circ} \mathrm{C}$ for $20 \mathrm{mins}$.

\subsection{Staining of Films Using Giemsa Stain}

$3 \%(1: 3)$ dilution of the Giemsa stain was made in buffered water ( $\mathrm{pH}$ 7.1-7.2) immediately before staining and the films were stained using the following procedure as described by WHO [5].

The slides were placed on a staining rack and flooded with $3 \%$ Giemsa stain and stained for 45 minutes. The slides were washed gently and thoroughly with buffered water $\mathrm{pH}$ 7.2, the back wiped and air dried in a vertical position. When dried, the slides were mounted with DPS mountant.

\subsection{Counting of Parasite Density in Blood}

The estimated number of parasites in a microliter $(\mu 1)$ of blood was counted using WHO method of counting parasite density in thick film [5]. The number of parasites counted alongside the count of 8000 leukocytes (WBC) is equivalent to the number of parasites in one microliter of blood [5]. Using two tally counters (one for malaria parasite, one for leukocytes), the number of parasites alongside $200 \mathrm{WBC}$ were recorded. The number of parasites in $8000 \mathrm{WBC}$ was calculated and recorded as the number of parasites $/ \mu 1$ of blood. If after the count of $200 \mathrm{WBC}$, less than 100 parasites were counted alongside, the counting was continued up to $500 \mathrm{WBC}$.

\subsection{Performing the RDTs for Detection of Malaria in Whole Blood Using the Four RDTs Under Review}

Each of the sample examined microscopically in which the malaria parasite density is estimated was also tested for the detection of malaria antigen using each of the four RDTs (SD Bioline, Global Device, LabAcon and CareStart). The tests were done the same day of sample collection, and according to the manufacturer's guidelines and procedures.

\subsection{Statistical Analysis}

Statistical analysis was done using appropriate medical software (Epi Info 7.2). Chi Square was conducted to determine the significant difference between the performance of the four diagnostic test kit and that of the conventional method. 


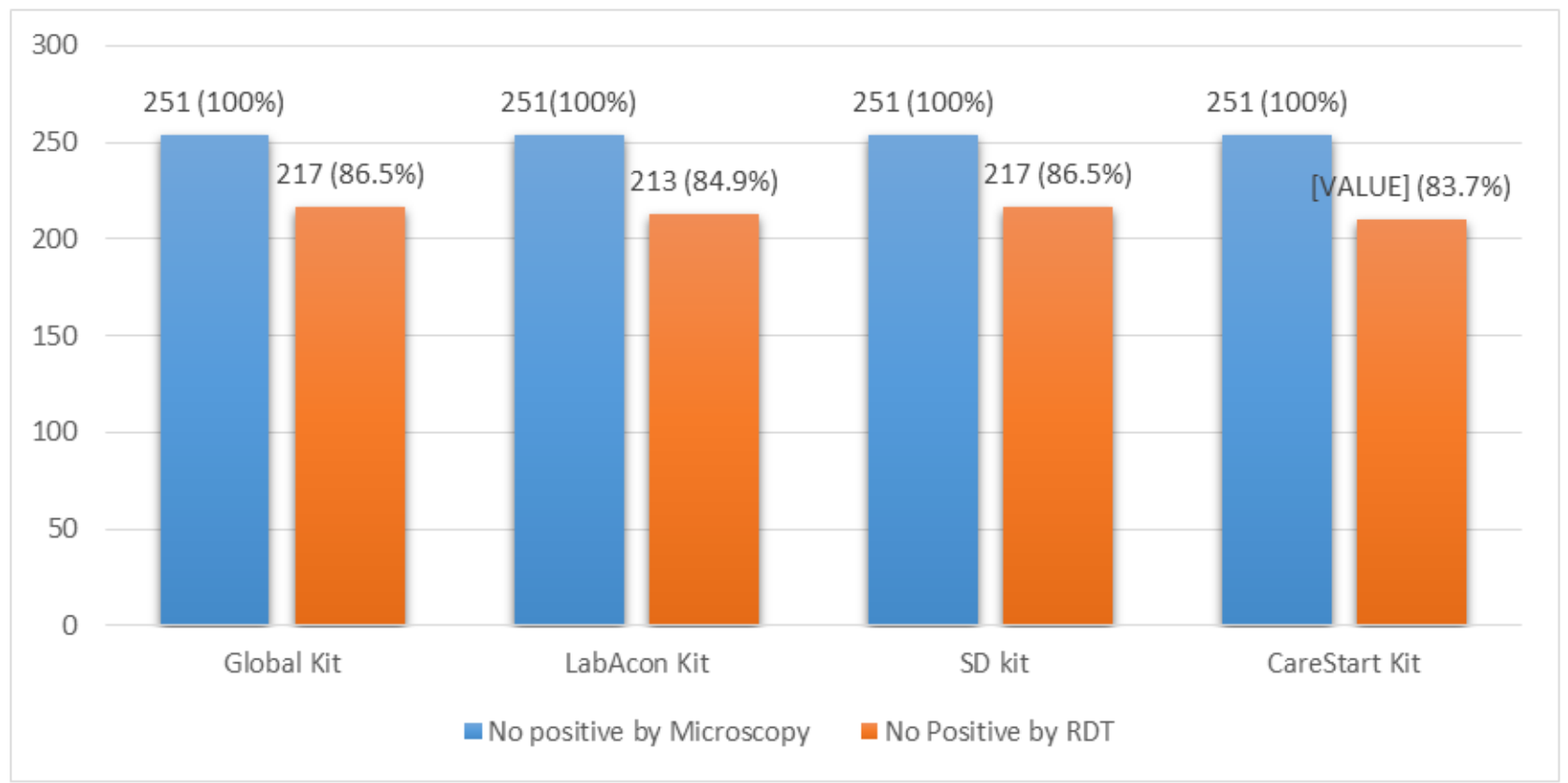

Figure 1. Sensitivity of four Rapid Test Kits used in the study.

\section{Results}

\subsection{Sensitivity of Four Rapid Test Kits Used in the Study}

Figure 1 reveals the sensitivity of the four rapid test kits. Global kit and SD Bioline had a sensitivity of $86.50 \%$ with $95 \%$ confidence interval of $80.70-90.10 \%$. LabAcon kit had a sensitivity of $84.90 \%$ with $95 \%$ confidence interval of 78.70 $88.60 \%$. CareStart kit had a sensitivity of $83.70 \%$ with $95 \%$ confidence interval of $77.30-87.50 \%$.

\subsection{Specificity of Four Rapid Diagnostic Kits Used in the Study}

Figure 2 presents the specificity of the four rapid test kits. CareStart kit had the highest sensitivity of $96.00 \%$ with $95 \%$ confidence interval of $92.10-97.10 \%$. SD Bioline kit had a specificity of $95.80 \%$ with $95 \%$ confidence interval of 91.70 $96.80 \%$. Global kit and LabAcon had a specificity of $95.30 \%$ with $95 \%$ confidence interval of 91.70-95.80.

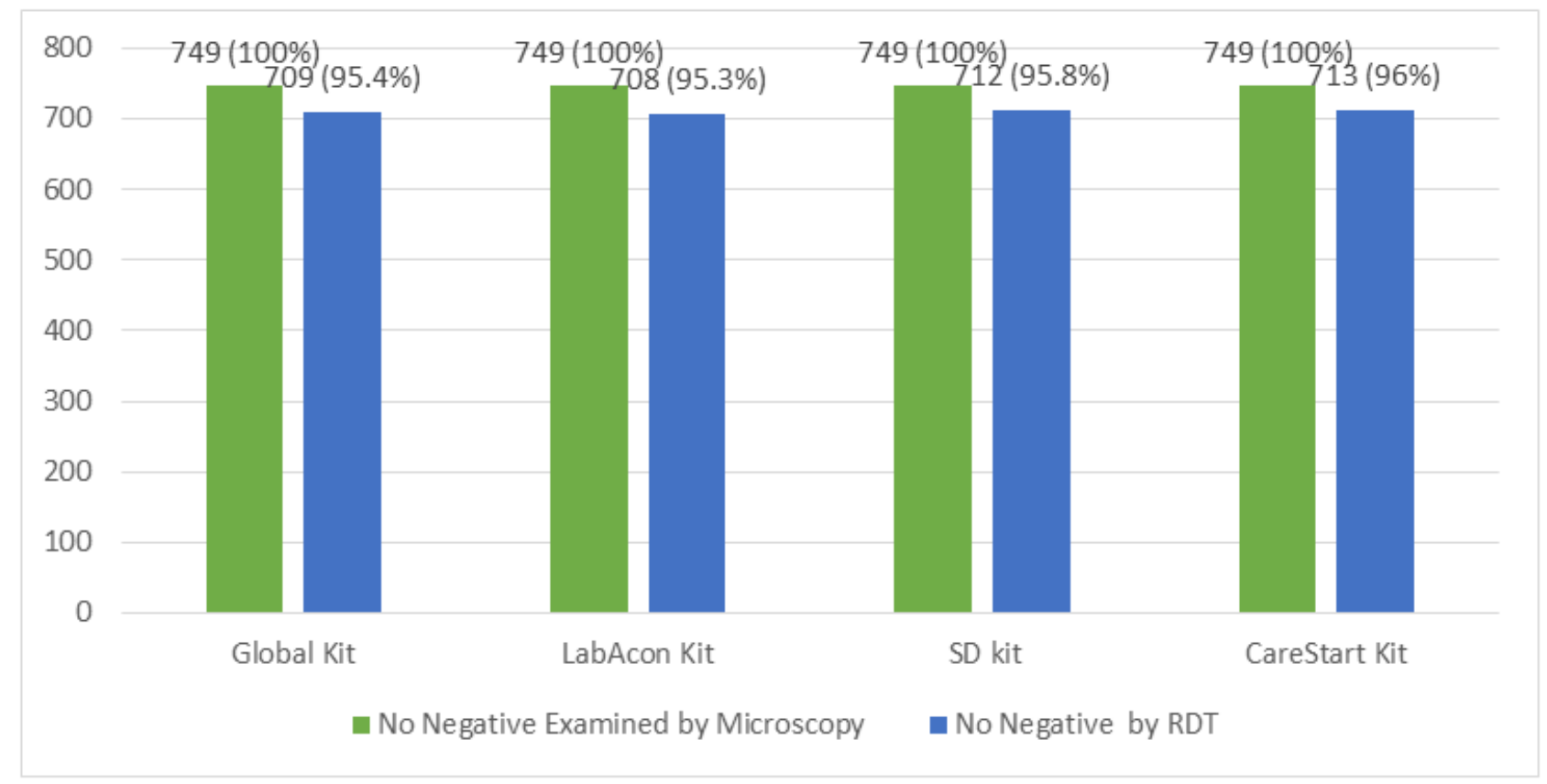

Figure 2. Specificity of four rapid diagnostic kits used in the study. 


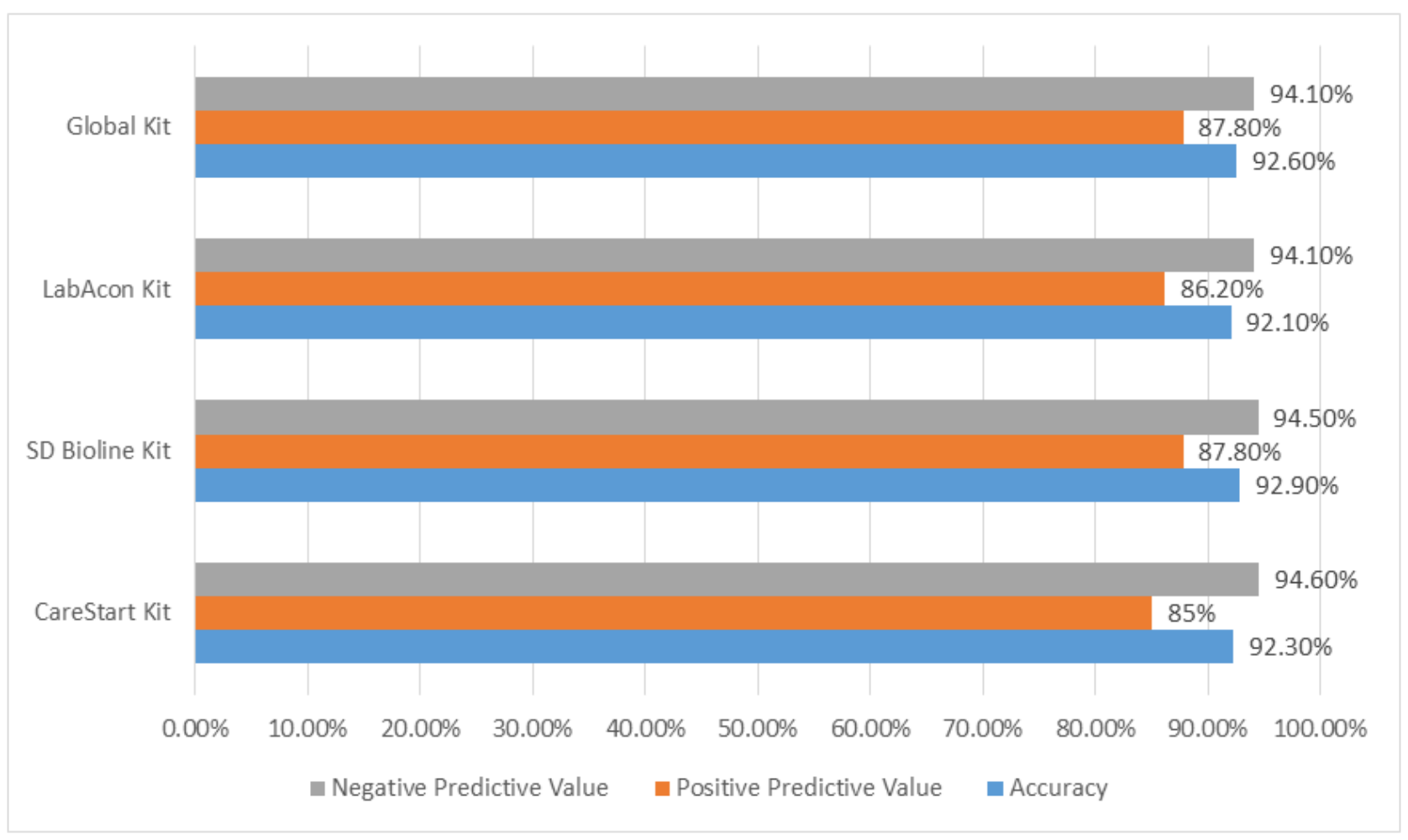

Figure 3. Accuracy, Positive Predictive Value and Negative Predictive Value of the Rapid Test Kits.

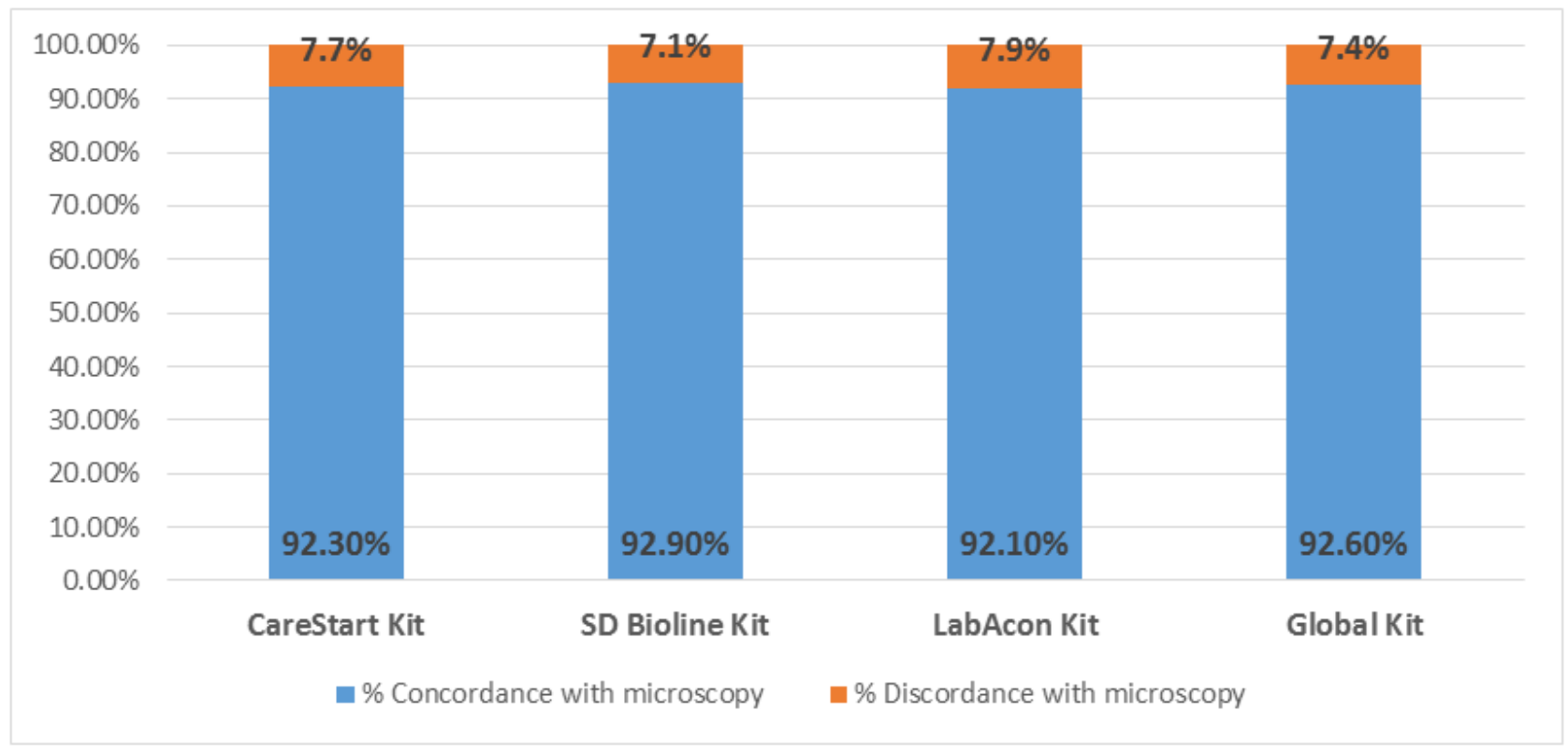

Figure 4. Percentage concordance of the four rapid diagnostic kits relative to microscopy examination.

\subsection{Accuracy, Positive Predictive Value and Negative Predictive Value of the Rapid Test Kits}

Figure 3 shows the accuracy, positive predictive value and negative predictive value of the rapid test kits. SD Bioline had the highest accuracy $(92.90 \%)$ followed by Global Kit (92.60\%), CareStart (92.30\%) and LabAcon (92.10\%). In terms of positive predictive value, Global Kit and SD Bioline had the same performance $(87.80 \%)$, LabAcon $(86.20 \%)$ and CareStart had $85.00 \%$. In terms of negative predictive value,
CareStart had the highest (94.60\%), SD Bioline (94.50\%), while Global Kit and LabAcon had the same performance (94.10\%). Cross tabulation on accuracy, positive predictive value and negative predictive value were not statistically significant $(\mathrm{p}>0.05)$.

\subsection{Percentage Concordance of the Four Rapid Diagnostic Kits Relative to Microscopy Examination}

Figure 4 presents percentage concordance of the four rapid diagnostic kits relative to microscopy as the gold standard. 
SD Bioline had the highest degree of concordance (92.90\%), followed by Global Kit (92.60\%), CareStart (92.30\%) and LabAcon $(92.10 \%)$. Cross tabulation among the RDTs was not statistically significant (Chi square $=0.108 ; \mathrm{p}=0.74$ ). However, cross tabulation relative to microscopy against each RDT showed statistically significant differences (Global kit $\mathrm{p}<0.0001$; SD Bioline $\mathrm{p}<0.0001$; LabAcon $\mathrm{p}<0.0001$ and CareStart $\mathrm{p}<0.0001)$.

\section{Discussion}

The test kits under review are the ones that are used locally to diagnose malaria in Nigeria. All the RDT kits detect the HRP2 which is expressed only by $P$. falciparum. Hence only the samples that were positive for $P$. falciparum were used to determine their sensitivity. Sensitivity and specificity of the four malaria RDT Kits-Global, LabAcon, SD Bioline and CareStart were compared in this work with microscopy as a standard. Accordingly, the sensitivity of the RDT kits were, $86.5 \%, 84.9 \%, 86.5 \%$ and $83.7 \%$ for Global, LabAcon, SD Bioline and CareStart respectively, while their specificity was 95.4\%, 95.3\%, 95.8\% and 96.0\% for Global, LabAcon, SD Bioline and CareStart respectively. This finding is similar to earlier report of Maltha et al. (2010) [6], who demonstrated that CareStart had a sensitivity range of $84.8 \%-92 \%$. However, this finding did not agree with the finding of Djalle et al. (2014) [7] who reported that SD Bioline had a sensitivity range of $88.1-95.4 \%$ and specificity range of $76.2-87.2 \%$, though they are not wide apart. Conversely, the finding of this work also disagrees with that of Gasser et al., (2005) [8], that BinaxNow, another HRP2 detecting RDT, had a sensitivity of $95 \%$ in overall assessment. Additionally, the results further revealed a slight difference from that of Pieroni et al., (1998) [9], who investigated the sensitivity and specificity of ParaSight F, another HRP" detecting RDT and demonstrated a sensitivity and specificity of 88 and $97 \%$ respectively though they are similar. Reasons for this slight variations in sensitivity and specificity of the RDTs could possibly be the stage of the parasite, the quantity of HRP2 antigen expressed by the parasite, structure variation of the antigen and host immunity [10]. Similar to the work of Djalle et al (2014) [7], and Wongsrichanalai et al (1991) [11], this work showed that sensitivity and test band of the test kits under review correlated directly with the parasite density. At parasitaemia of 126 parasites $/ \mu 1$, all the test kits gave positive result, hence a sensitivity of $100 \%$ at that parasite density. This Lowest Level of Detection (LLD) of the test kits under review is far higher than the WHO recommended LLD of 200 parasites/ $\mu 1$ [10]. The study showed that SD Bioline, Global and LabAcon detected parasitaemia level as low as 110 parasites/ $\mu 1$ but CareStart could not. This finding gave the other kits an edge over CareStart. Clinically, however, a parasitaemia of 110 parasites/ $\mu \mathrm{l}$ can be said to be subclinical level [12]. This suggests that the kits can comfortably be deployed for diagnosis of symptomatic Plasmodium falciparum infection. However, since the undetectable level of parasitaemia will persist and elicit pathogenic effect overtime and also be transmitted, this method of diagnosis cannot replace microscopy. At parasitaemia level lower than 110 parasites/ $\mu 1$, none of the kits under review was able to detect malaria parasite. Therefore, result in this work showed that microscopy detected parasite density as low as 64 parasites/ $\mu$, affirming the fact that it remains the gold standard in malaria diagnosis particularly in low level infection and when it is done by some well trained personnel.

Accuracy is the degree of concordance with the standard. This is a function of the positive predictive value (PPV) and negative predictive value (NPV). Positive predictive value of a diagnostic test is the probability that the disease is present in a patient with a positive test result while negative predictive value is the probability that the disease is not present in a patient with a negative test result. The finding from this study showed that SD Bioline and Global had PPV of $87.8 \%$ while LabAcon and CareStart had PPV of $86.2 \%$ and $85 \%$ respectively. While CareStart had the highest NPV of $94.6 \%$, SD Bioline had that of $94.5 \%$ and LabAcon and Global each had a NPV of $94 \%$ respectively. The PPV of all the kits in this study were superior to that of Elizabeth et al., (2016) [13] who demonstrated a PPV of $62 \%$ for SD Bioline but the NPV in her work is similar to report of this study. The high level of NPV of the test kits under review in this work suggests that the kits will perform better in predicting negative $P$. falciparum infection. Many sample (32) were negative with microscopy but positive with the kits. These patients might not have active $P$. falciparum infection, the parasites having been cleared with appropriate treatment but the HRP2 still persisted in the blood and so was detected as similarly recorded by Oguonu et al (2014) [3]. All the 32 samples negative with microscopy show very faint test bands suggesting that the circulating HRP2 were clearing from the system. From this study, the percentage concordance of the test kits relative to microscopy is $92.90 \%$ for SD Bioline, 92.60\% for Global, $92.30 \%$ for CareStart and $92.10 \%$ for LabAcon. All the four kits had relatively high level of performance, but comparison with each other, showed that there is no statistical difference $(\mathrm{P}>0.005 / 0.001)$. However, cross tabulation of each kit against microscopy, showed a statistical difference $(\mathrm{P}>0.005 / 0.001)$ in performance proving that microscopy is superior to the RDTs hence cannot be replaced with RDT. The RDTs therefore, need to be improved upon for more efficiency.

\section{Conclusion}

The study compared the accuracy of four RDTs (Care start, SD-Bioline, LabAcon and Global) with the traditional method of microscopy.

CareStart and SD Bioline are WHO prequalified and recommended RDT kits and hence adopted by the National Program on Malaria Control. Though the other two are not recommended by the National Program on Malaria Control, the sensitivity, specificity, positive predictive value, negative predictive value and accuracy of the four kits were not statistically different. Therefore, program managers may 
wish to incorporate any or both of them into the list of RDTs approved for use in Nigeria and also import and distribute them to public health facilities in the country like CareStart and SD Bioline.

There is significant difference, however, between the performance of each of the four kits and microscopy. Therefore, RDTs have important role to play in Nigeria malaria control and elimination, but they cannot comfortably and completely replace microscopy. The LLD of the RDTs were very low (110 parasites $/ \mu$ for SD Bioline, Global and LabAcon and 125 parasites $/ \mu$ for CareStart). This parasitaemia is subclinical level of malaria infection, hence the performance of the RDTs under study were satisfactory for routine diagnostic use especially in rural setting lacking electricity and manpower for microscopy.

\section{References}

[1] Moody, A. (2002): Rapid diagnostic test malaria parasites. Clinical Microbiology Review, 15: 66-78.

[2] Harchut, K., Standly, C., Dobson, A., Klaasen, B., RambaudAlthaus, C., Althaus, F. and Nowak, K., (2013). Overdiagnosis of malaria by microscopy in the Kilombero valley, Southern Tanzania: an evaluation of the utility and cost effectiveness of the rapid diagnostic tests. Malar J., 12: 159$10.1186 / 1475-2875-12-159$.

[3] Oguonu, T., Shu, E., Ezeonwu, B. U., Lige, B., Derrick, A., Umeh, R. E. and Agbo, E. (2014). The performance evaluation of a urine malaria test (UMT) kit for the diagnosis of malaria in individuals with fever in south-east Nigeria: cross-sectional analytical study. Malaria Journal. 13: 403.

[4] Chesbrough, M. (2005). District Laboratory Practice in Tropical Countries Part 1. Second edition. Cambridge low priced editions. Pp 239-258.

[5] WHO (2004). Manual of basic techniques for a health laboratory. $\mathrm{Pp}$ 179-182.
[6] Maltha, J., Gillet, P., Bottieau, E., Cnops, L., van Esbroeck, M. and Jacobs, J. (2010). Evaluation of a rapid diagnostic test (CareStart Malaria HRP-2/pLDH (Pf/pan) Combo Test) for the diagnosis of malaria in a reference setting. Malar J.; 9: 171 .

[7] Djalle, D., Gody, J. C., Moyen, J. M., Tekpa, G., Ipero, J. and Madji, N. (2014). Performance of Paracheck-Pf, SD Bioline malaria Ag-Pf and SD Bioline malaria Ag-Pf/pan for diagnosis of falciparum malaria in the Central African Republic. BMC Infect Dis.; 14: 109.

[8] Gasser, R. A., Ruebush, T. K., Miller, R. S., Sirichaisinthop, J., Forney, J. R. and Bautista, C. T. (2005). Malaria diagnosis: Performance of NOW ICT malaria in large scale field trial. AJTMH; 54th Annual Meeting.

[9] Pieroni, P., Mills, C. D., Ohrt, C., Harrington, M. A. and Kain, K. C. (1998). Comparison of the ParaSight F and the ICT malaria Pf test with the polymerase chain reaction for the diagnosis of malaria in travelers. Trans. R. Soc. Trop. Med. Hyg. 92: 166-169.

[10] WHO (2004a). WHO Informal consultation on Laboratory Methods for Quality Assurance of malaria Rapid Diagnostic Tests. WHO Regional Office for Western Pacific. Manila, Philippines, 20-22 July, 2004.

[11] Wongsrichanalai, C., Pornsilapatip, J., Namsiriponpun, V., Pansamdang and Wilde, H. (1991). Acridine orange fluorescent microscopy and the detection of malaria in population with low density parasitemia. Am. J. Trop. Med. Hyg. 44: 17-20.

[12] Daniel, M. P., Rupam, T., Thomas, J. P., Richard, J. M., Chea, N., Jeremy, C., Pasathorn, S., Mallika, I., Lorenz von, S., Nicholas, J. W. and Arjan, M. D (2017). A multi- level spartial analysis of clinical malaria and subclinical Plasmodium infections in Pailin Province, Cambodia. Heliyon; 3 (11) e00447.

[13] Elizabeth, W. W., Nickline, K., Collins, M., Mark, H., Jacob, D. J., Carolyne, M., Lalaine, A., Bernhards, O. and Colin, O. (2016). Field evaluation of diagnostic performance of malaria rapid diagnostic tests in western Kenya. Malaria Journal. 15: 456. 